\title{
Floating-Potential Dielectrophoresis-Controlled Fabrication of Single-Carbon-Nanotube Transistors and Their Electrical Properties
}

\author{
Lifeng Dong, ${ }^{\dagger}$ Vachara Chirayos, ${ }^{\dagger}$ Jocelyn Bush, ${ }^{\dagger}$ Jun Jiao, ${ }^{*}, \dagger$ Valery M. Dubin,, \\ Ramanan V. Chebian, ${ }^{\ddagger}$ Yoshi Ono, ${ }^{\S}$ John F. Conley, Jr., ${ }^{\S}$ and Bruce D. Ulrich ${ }^{\S}$ \\ Department of Physics, Portland State University, Portland, Oregon 97207-0751, Portland Technology \\ Development, Intel Corporation, Hillsboro, Oregon 97124, and Integrated Circuit Process Technology Group, \\ Sharp Labs of America, Camas, Washington 98607
}

Received: April 7, 2005; In Final Form: May 18, 2005

\begin{abstract}
We present a floating-potential dielectrophoresis method used for the first time to achieve controlled alignment of an individual semiconducting or metallic single-walled carbon nanotube (SWCNT) between two electrical contacts with high repeatability. This result is significantly different from previous reports, in which bundles of SWCNTs were aligned between electrode arrays by a conventional dielectrophoresis process where the results were only collected from the control electrode regions. In this study, our alignment focus is not only on the regions of the control electrodes but also on those of the floating electrodes. Our results indicate that bundles of carbon nanotubes along with impurities were first moved into the region between two control electrodes while individual nanotubes without impurities were straightened and aligned between two floating electrodes. The measurements for the back-gated nanotube transistors made by this method displayed an on-off ratio and transconductance of $10^{5}$ and $0.3 \mu \mathrm{S}$, respectively. These output and transport properties are comparable with those of nanotube transistors made by other methods. Most importantly, the findings in this study show an effective way to separate individual nanotubes from bundles and impurities and advance the processes for site-selective fabrication of single-SWCNT transistors and related electrical devices.
\end{abstract}

\section{Introduction}

Due to their unique electrical properties, single-walled carbon nanotubes (SWCNTs) have been extensively studied as the building blocks for nanoscale electronic devices. ${ }^{1-5}$ However, as-synthesized SWCNTs are in the form of bundles with other impurities such as catalyst particles and amorphous carbon debris; therefore, an effective method to disperse, purify, and display them on specified locations for device fabrication has to be developed. In addition, it is critical to be able to selectively deposit both semiconducting and metallic individual nanotubes for different electronic applications, such as field effect transistors (FET) and interconnects. Although a worldwide effort has been carried out by a number of groups, further research is needed to develop a reliable and comprehensive nanofabrication technique for SWCNT applications. Recently, several groups reported that, using alternating current (AC) dielectrophoresis, metallic nanotubes could be selectively separated from admixtures of metallic and semiconducting nanotubes and then aligned onto a microelectrode array. ${ }^{6-12}$ However, no effective methods have been developed, to date, on positioning an individual SWCNT between two electrical contacts by AC dielectrophoresis. In this paper, we report an effective dielectrophoresis process that uses a combination of control electrodes and floating electrodes to successfully place individual carbon nanotubes onto specified electrodes with high repeatability. Through the use of this process, individual semiconducting or metallic nanotubes are aligned between the floating electrodes, while bundles of

* Author to whom correspondence should be addressed. Phone: (503) 725-4228. Fax: (503) 725-9525. E-mail: jiaoj@pdx.edu.

† Portland State University.

$\doteqdot$ Intel Corporation.

$\S$ Sharp Laboratories of America.
SWCNTs and the impurities were attracted onto control electrodes. During this study, to evaluate the effectiveness of this process, we utilized various electron microscopy techniques to map the location of aligned nanotubes and to characterize their morphology, internal structure, and elemental composition. Furthermore, a number of single-SWCNT transistors were fabricated and characterized. Nanotube transistors fabricated by this technique appeared to exhibit excellent electrical properties in terms of steep subthreshold slope and high transconductance.

\section{Experimental Methods}

Dielectrophoresis Process for Dispersion and Alignment of SWCNTs. To date, all of the reports on dielectrophoresis alignments of SWCNTs have only focused on the investigation of the regions between two control electrodes or two parallel electrode arrays where metallic nanotubes or bundles mixed with metallic and semiconducting nanotubes were deposited. Occasionally, an individual metallic nanotube was found between two electrodes. ${ }^{12}$ For the fabrication of SWCNT electronics, a reliable technique needs to be explored to effectively align individual nanotubes between two electrodes, especially single semiconducting nanotubes. To achieve the alignment of an individual nanotube and study the influence of surrounding floating potentials on the nanotube alignment, a series of electrodes with two different kinds of geometries (rectangular and triangular) and with spacing ranging from $0.5 \mu \mathrm{m}$ to 2.5 $\mu \mathrm{m}$ were designed, as shown in Figures 1 and 2. The electrodes were fabricated on a silicon substrate covered with a thermal oxide layer (1- $\mu \mathrm{m}$-thick) by a standard photolithography method. To separate bundles of carbon nanotubes into individual tubes, we used a similar dispersion method as reported by O'Connell 


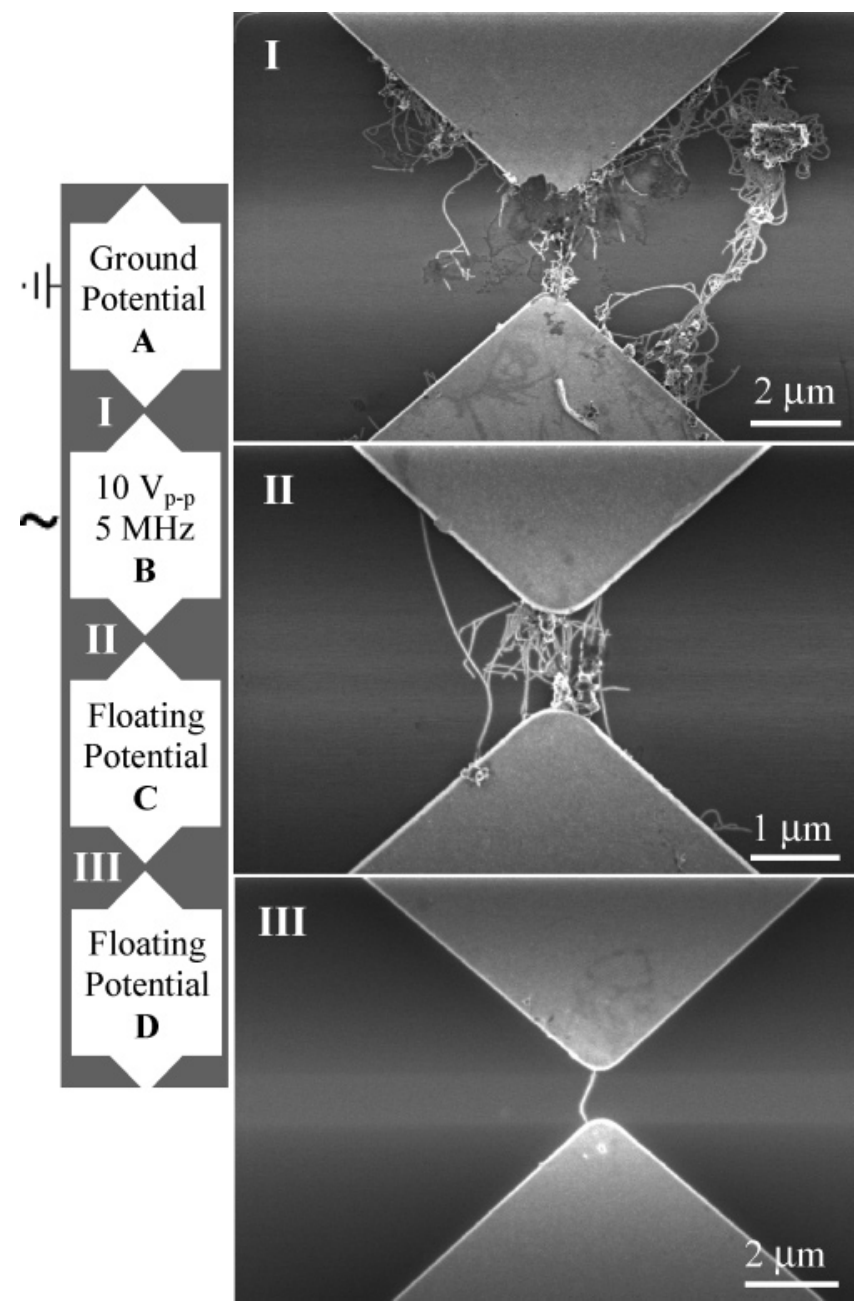

Figure 1. Schematic diagram of the combination layout of the control electrodes (A and B) and floating electrodes (C and D) used in the dielectrophoresis experiments and a typical set of SEM images of the nanotubes varying in numbers deposited onto the electrode regions of I, II, and III.

et al. ${ }^{13}$ In our experiments, a trace amount of SWCNT powder (BuckyUSA Company) was first dispersed in deionized water containing 1 wt \% sodium dodecyl sulfate (SDS). The suspension was then sonicated for $10 \mathrm{~min}$ and centrifuged at $120000 \mathrm{~g}$ for $30 \mathrm{~min}$. After centrifugation, the upper $95 \%$ of the clean supernatant was decanted for dielectrophoresis experiments.

During the dielectrophoresis experiments, $1.5 \mu \mathrm{L}$ of the nanotube suspension described above was dropped onto a selected substrate region containing two control electrodes (one of them grounded and the other applied with a sinusoidal potential) and several floating electrodes. As suggested by its name, during the dielectrophoresis process, the electrical potential of a floating electrode is of a floating instead of a fixed magnitude. In the case of nanotube alignment, the potential magnitude depends on (1) the conductivity of the nanotube suspension, (2) the density of nanotubes deposited on the region between the two control electrodes, and (3) the type of deposited nanotubes (semiconducting or metallic). The details are further discussed in the next section.

Although a general dielectrophoresis experiment could be carried out using either direct current (DC) or AC electrical fields. ${ }^{14,15} \mathrm{AC}$ dielectrophoresis has been reported to be more effective for the alignment of SWCNTs, with optimum frequencies reported to be between 2 and $10 \mathrm{MHz}^{6-12}$ In our experiments, we investigated an extended frequency range. It
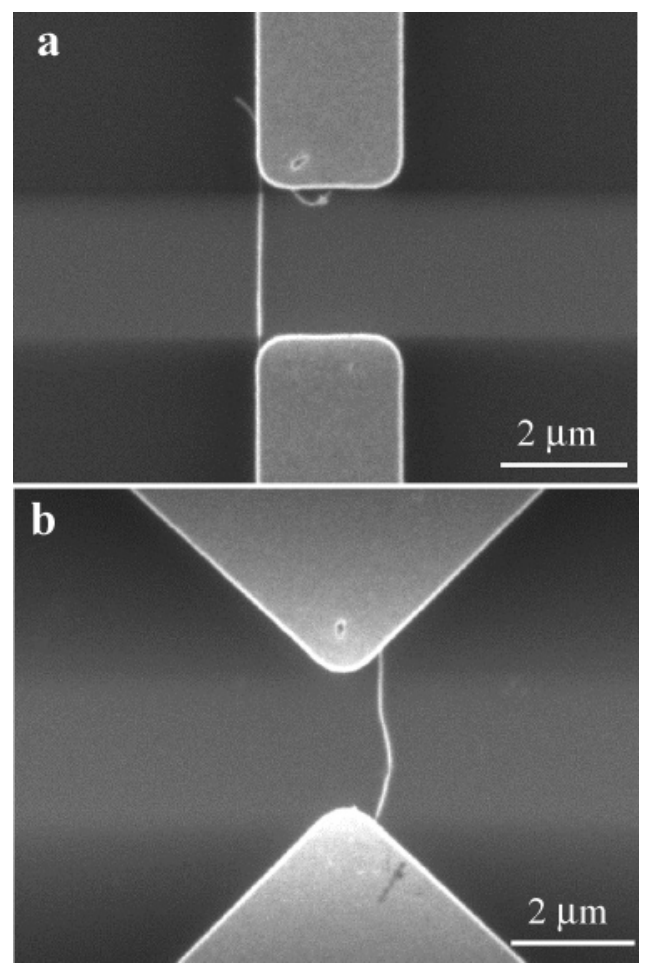

Figure 2. SEM images of one individual nanotube without other impurities aligned between two floating electrodes with two kinds of geometry: (a) rectangular and (b) triangular. The longitudinal axis of the nanotubes tends to orient along electric field directions.

was found that at a frequency lower than $50 \mathrm{kHz}$ electrohydrolysis occurred and bubbles were formed on the surfaces of the electrodes. This reaction eventually destroyed the electrodes. When an electric field of $10 \mathrm{~V}_{\mathrm{p}-\mathrm{p}}$ and a frequency of $5 \mathrm{MHz}$ were applied between the control electrodes, well-aligned individual SWCNTs were observed between a number of paired floating electrodes. To prevent the presence of SDS molecules on the surfaces of the nanotubes after dielectrophoresis and to eliminate their influence on the electrical properties of the aligned nanotubes, the substrates with aligned nanotubes were then soaked in deionized water for 15-20 min, rinsed with deionized water, and dried with flowing nitrogen gas.

Electron Microscopy Characterization of Aligned SWCNTs. A FEI Sirion field emission scanning electron microscope (SEM) was utilized to characterize the morphology and the orientation of individual nanotubes aligned between two electrodes. We also used the SEM to map the location of the aligned nanotubes having good contact with electrodes for later electrical measurements. To minimize the influence of the electron beam on the aligned SWCNTs, the acceleration voltage of the SEM was operated at 1 or $2 \mathrm{kV}$.

A FEI Tecnai F20 high-resolution transmission electron microscope (TEM) was employed to study the effectiveness of the nanotube dispersion and separation assisted by SDS molecules and the integrity of the nanotube structures after the SDS dispersion process. An energy-dispersive X-ray (EDX) spectrometer attached to the TEM was used to analyze the elemental composition of the nanotubes after the cleaning process of the SDS molecules. To prepare the TEM sample, 5 $\mu \mathrm{L}$ of the as-prepared SDS-SWCNTs suspension was dropped onto a TEM copper grid coated with a holey carbon thin film. The grid was soaked in deionized water for $30 \mathrm{~min}$ and then dried naturally. This is similar to the cleaning process applied to the silicon substrate containing aligned SWCNTs after the dielectrophoresis experiment. 
Electrical Characterization. According to SEM-mapped positions on the photolithography-generated electrode patterns, electrodes aligned with individual SWCNTs were accurately located under the probe station. Electrical measurements were carried out on an individual nanotube instead of bundles. Since our electrodes were made of platinum and deposited on the thermal oxide layer of a silicon wafer, they were used directly as the source and drain for an individual nanotube transistor aligned between them. For this type of carbon-nanotube transistor, the silicon substrate was connected as the back-gate, and no other metal was deposited on top of the nanotubes after the dielectrophoresis. The source-drain current $\left(I_{\mathrm{ds}}\right)$ through the nanotubes as a function of the bias voltage $\left(V_{\mathrm{ds}}\right)$ and the gate voltage $\left(V_{\mathrm{gs}}\right)$ was measured at room temperature using standard DC techniques with a HP 4145B semiconductor parameter analyzer.

\section{Results and Discussion}

Dielectrophoresis Separation and Site-Selective Positioning. To explain why the individual nanotubes were found in the floating electrode areas while the bundles and most of the impurities were attracted to the control electrodes, one should first understand the effect of the electrical field on the materials processed. It is well-understood that when uncharged dielectric or conductive matter is placed in an electric field the electric field will induce the formation of a dipole moment inside the matter. If the field is uniform, then the net Coulombic force on the dipole is zero. Otherwise, in a nonuniform electric field, the net Coulombic force is not zero. This can be depicted in eq 1 , where $\overrightarrow{\mathbf{F}}$ is the net force, $\overrightarrow{\mathbf{E}}$ is the electric field, and $\vec{P}$ is the induced dipole moment. In this case, the electric field is simply expanded about the position $\overrightarrow{\mathbf{r}}$ using a vector Taylor series expansion. ${ }^{14,15}$

$$
\begin{gathered}
\overrightarrow{\mathbf{F}}=q \overrightarrow{\mathbf{E}}_{(\overrightarrow{\mathbf{r}}+\overrightarrow{\mathbf{d}})}-q \overrightarrow{\mathbf{E}}_{(\overrightarrow{\mathbf{r}})} \\
=q\left(\overrightarrow{\mathbf{E}}_{(\overrightarrow{\mathbf{r}})}+\overrightarrow{\mathbf{d}} \cdot \nabla \overrightarrow{\mathbf{E}}_{(\overrightarrow{\mathbf{r}})}+\cdots\right)-q \overrightarrow{\mathbf{E}}_{(\overrightarrow{\mathbf{r}})} \\
=q \overrightarrow{\mathbf{d}} \cdot \nabla \overrightarrow{\mathbf{E}}_{(\overrightarrow{\mathbf{r}})}+\cdots \\
\approx q \overrightarrow{\mathbf{d}} \cdot \nabla \overrightarrow{\mathbf{E}}_{(\overrightarrow{\mathbf{r}})} \\
=\overrightarrow{\mathbf{P}} \cdot \nabla \overrightarrow{\mathbf{E}}_{(\overrightarrow{\mathbf{r}})}
\end{gathered}
$$

Since the net force is not zero, a polarizable object could be moved around in a nonuniform electric field. Notice that in eq 1 the magnitude of the force $\overrightarrow{\mathbf{F}}$ is not proportional to the magnitude of $\overrightarrow{\mathbf{E}}$ but is directly related to the gradient of the electrical field $\nabla \overrightarrow{\mathbf{E}}$. This means that the dielectrophoretic alignment of nanotubes does not just depend on the magnitude of the electrical field but on the electrical field gradient $\nabla \overrightarrow{\mathbf{E}}$. This suggests that we should not only pay attention to the alignment of nanotubes between two control electrodes (location I to the left of Figure 1) as reported in the literature ${ }^{6-12}$ but also look into the regions among floating electrodes (locations II and III). A set of representative SEM images taken from those three regions (Figure 1) indicates the distributions of nanotubes aligned at various locations. Note at location I, between two control electrodes (one was kept at ground potential and the other was applied with an AC potential of $10 \mathrm{~V}_{\mathrm{p}-\mathrm{p}}$ and 5 $\mathrm{MHz}$ ), that several bundles of nanotubes, amorphous carbon debris, and other impurities were clustered around the electrodes. However, at location II, fewer nanotubes with relatively high purity were deposited between the electrodes. At location III, a single nanotube without any other impurities was aligned between the two floating electrodes. We have applied

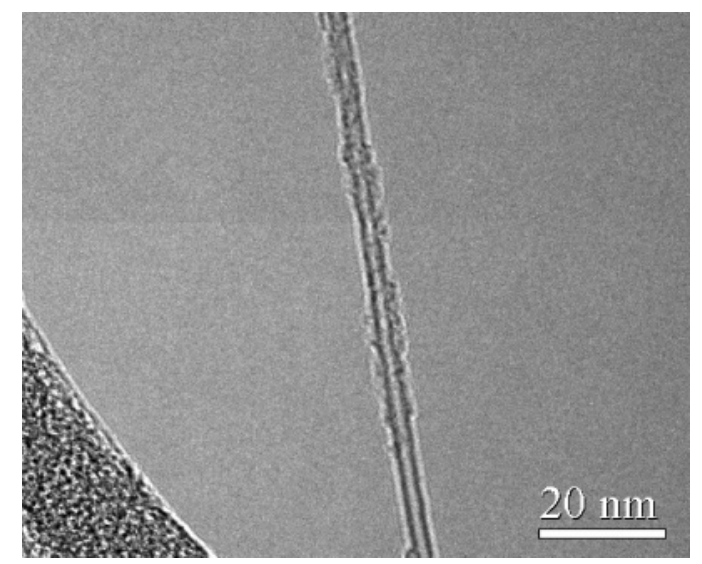

Figure 3. TEM image of an individual nanotube partially coated with some amorphous carbon. There was no signal of sodium and sulfate obtained from EDX analysis for the same nanotube. This indicates that SDS molecules not only can be used to separate nanotube bundles but also are removable after the dielectrophoresis alignment.

the nanotube suspension and AC field onto about 100 regions between two control electrodes and found 26 pairs of floating electrodes, each aligned with individual nanotubes. This represents a rate of $26 \%$ for the alignment of individual SWCNTs. As demonstrated in Figures $2 \mathrm{a}$ and $2 \mathrm{~b}$, we were able to align individual nanotubes between electrodes with either rectangular or triangular shape. Note in Figure 2a the image contrast of the two ends of a SWCNT is different from that of the central part of the tube, which could be caused by the fact that the nanotube ends are connected with conductive Pt electrode layers and the central part is located above the dielectric $\mathrm{SiO}_{2}$ layer. This phenomenon might also be explained using the two different mechanisms proposed by Brintlinger et al. and Homma et al., where they attempted to explain the imaging of the nanotubes on an insulator substrate with a bright contrast at low operation voltages. ${ }^{16,17}$

To examine the separation of nanotubes by SDS molecules and to investigate whether the SDS molecules could be cleaned from nanotubes after dielectrophoresis, TEM characterization was performed. Figure 3 shows a high-resolution TEM image of a well-separated SWCNT. Although the nanotube is partially covered by some amorphous carbon, a normal phenomenon for a SWCNT, no signal of sodium or sulfate was obtained from the EDX analysis of the same nanotube. This suggests that SDS molecules can be used to separate nanotubes and can be cleaned off from the nanotube surface by the procedures described in the previous section.

Dielectrophoresis is well-known for the manipulation of microspherical particles. ${ }^{14,15}$ Only recently, it has been used to manipulate elliptical objects, especially the one-dimensional nanotubes. ${ }^{6-12}$ However, systematic experimental results that could be explained by the existing theory seem lacking. Our results reported here, however, are qualitatively consistent with the theory developed for spherical particles, as described below. ${ }^{14,15}$ For a homogeneous and dielectric sphere, the dielectrophoresis force is given by

$$
\overrightarrow{\mathbf{F}}=2 \pi V \epsilon_{\mathrm{m}} K_{(\omega)} \nabla\left(\overrightarrow{\mathbf{E}}_{\mathrm{rms}}^{2}\right)
$$

where $V$ is the volume of the particle, $\epsilon_{\mathrm{m}}$ is the real part of the medium solution dielectric constant, $\overrightarrow{\mathbf{E}}_{\mathrm{rms}}$ is the root-mean-square value of the electric field, and $K$ is the Clausius-Mosotti factor, which depends on the particle dielectric constant $\epsilon_{\mathrm{P}}^{*}$ and medium solution dielectric constant $\epsilon_{\mathrm{m}}^{*}$ by 


$$
K_{(\omega)}=\operatorname{Re}\left(\frac{\epsilon_{\mathrm{P}}^{*}-\epsilon_{\mathrm{m}}^{*}}{\epsilon_{\mathrm{P}}^{*}+2 \epsilon_{\mathrm{m}}^{*}}\right)
$$

where $\epsilon^{*}$ is a complex quantity, which is related to its conductivity $\sigma$ and angular frequency $\omega$ by

$$
\epsilon^{*}=\epsilon-\mathrm{i} \frac{\sigma}{\omega}
$$

According to eqs 2-4, for a given suspension, the dielectrophoresis force is proportional to the volume of particles and depends on the electrical field gradient. On the basis of the above formulas, the positioning of an individual nanotube between two floating electrodes in Figure 1 can be explained as follows: When a droplet of nanotube suspension is added to the substrate consisting of two control electrodes and two floating electrodes, nanotube bundles, having a larger volume than individual nanotubes, experience a stronger dielectrophoretic force and are therefore deposited across the control electrodes (region I), where the largest electrical field gradient exists. In this case, nanotube bundles are considered as one suspension object. The low conductivity of the nanotube suspension (the resistivity of deionized water is $10 \mathrm{M} \Omega \mathrm{cm}$ ) causes the electrical potentials at electrodes $\mathrm{C}$ and $\mathrm{D}$ to be smaller than the potential at electrode B: $V_{\mathrm{B}} \gg V_{\mathrm{C}}, V_{\mathrm{C}}>V_{\mathrm{D}}$, and $V_{\mathrm{A}}=0$. In the meantime, other impurities larger than individual nanotubes are deposited at location I. Since the electrical field gradient at region II is larger than that at region III, some nanotube bundles and impurities are also aligned at location II, but the quantity is smaller than that at region I. After a majority of the nanotubes and impurities are attracted to regions I and II, there is a high probability for individual nanotubes to be deposited between floating electrodes $\mathrm{C}$ and $\mathrm{D}$ in location III, since an electrical field gradient exists in region III $\left(V_{\mathrm{C}}>V_{\mathrm{D}}\right)$. Our experimental results show that individual nanotubes were aligned at region III at a rate of $26 \%$. Therefore, with the use of a combination of control and floating electrode distributions, not only are individual nanotubes aligned between two electrical contacts, but bundles and other impurities are separated from individual nanotubes and trapped in the region of the control electrodes. Without the use of floating electrodes $\mathrm{C}$ and $\mathrm{D}$, an individual nanotube could be positioned between control electrodes A and B only if there were one or a few separated nanotubes in the suspension without other impurities. However, there has been no reported effective method to prepare a solution containing only one or a few nanotubes without other impurities. Besides, such a solution would not have much practical value.

During dielectrophoresis separation and site-selective positioning, the alignment of the nanotubes is not only controlled by the dielectrophoresis force but also by the torque $\overrightarrow{\mathbf{T}}$ exerted on the induced electrical dipole moment.

$$
\overrightarrow{\mathbf{T}}=\overrightarrow{\mathbf{P}} \times \overrightarrow{\mathbf{E}}=q \overrightarrow{\mathbf{d}} \times \overrightarrow{\mathbf{E}}
$$

Notice that the torque $\overrightarrow{\mathbf{T}}$ depends on the electric field vector $\overrightarrow{\mathbf{E}}$, not the electric field gradient $\nabla \overrightarrow{\mathbf{E}}$. Thus, in an electric field, the torque aligns the longitudinal axis of a nanotube to be parallel to the direction of the electric field, since when $\overrightarrow{\mathbf{d}}$ is parallel to $\overrightarrow{\mathbf{E}}, \overrightarrow{\mathbf{T}}$ is equal to zero. As shown in Figure 2, the longitudinal axes of individual nanotubes were oriented along electric field directions. Most of the well-aligned nanotubes were also straightened between electrodes as demonstrated in Figure 2a.

Electrical Characterization. Single-carbon-nanotube transistors were fabricated based on the configuration demonstrated
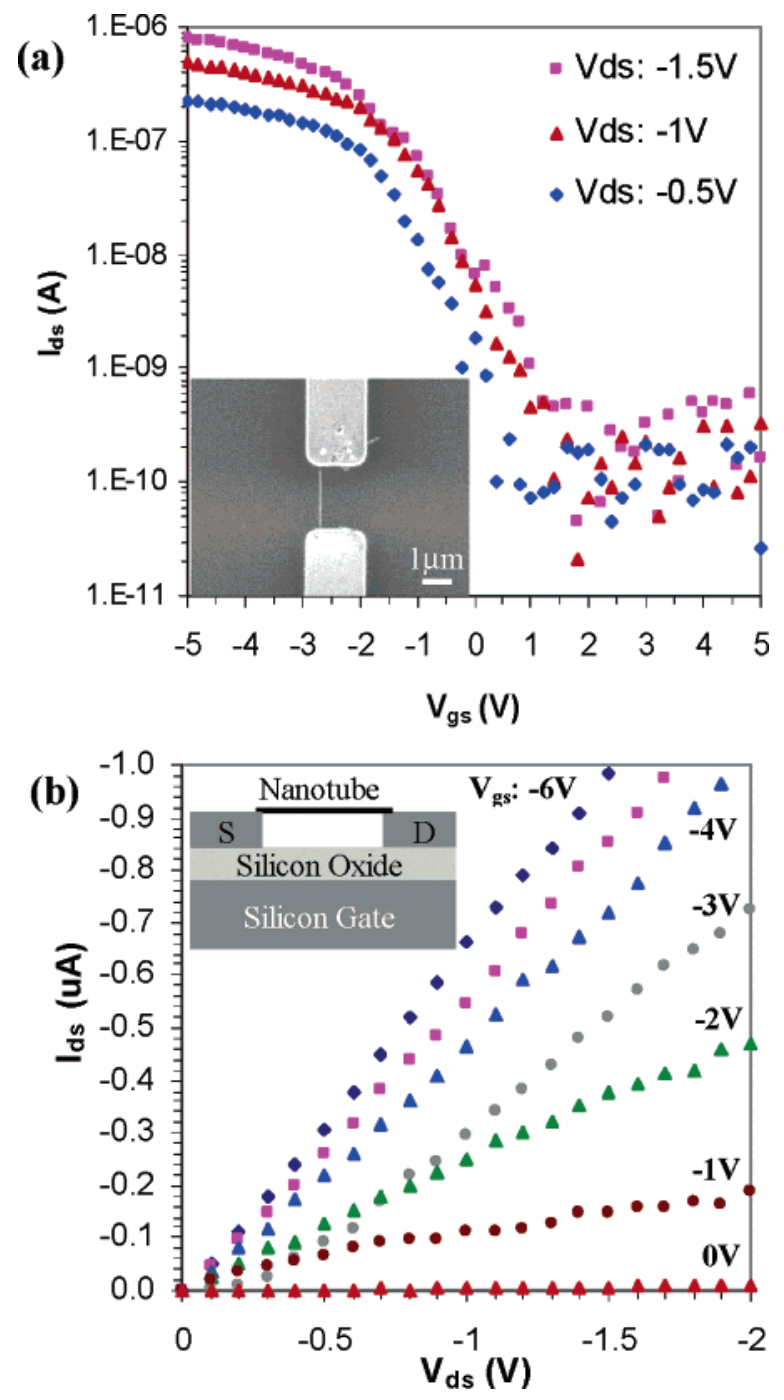

Figure 4. (a) Transport properties of a typical p-type nanotube transistor at the source-drain voltages of $-1.5,-1$, and $-0.5 \mathrm{~V}$, respectively. (Inset: SEM image of the nanotube positioned between two platinum electrodes.) (b) Corresponding gate-dependent $I_{\mathrm{ds}}$ vs $V_{\mathrm{ds}}$ behavior at the gate voltages from -6 to $0 \mathrm{~V}$. (Inset: Illustration of the back-gated nanotube transistor.) The source-drain separation is $2.5 \mu \mathrm{m}$, and the thickness of silicon oxide is $1 \mu \mathrm{m}$.

in Figure 2, where an individual nanotube was positioned between two platinum electrodes that directly serve as the source and drain for a nanotube transistor, as illustrated in the inset of Figure 4b. Electrical characterization of these fabricated nanotube transistors not only allowed us to study their output and transfer characteristics but also helped us to identify whether semiconducting or metallic nanotubes could be dielectrophoretically aligned between two electrical contacts. Our electrical measurements suggest (as shown in Figures 4 and 5) that both individual semiconducting and metallic nanotubes can be positioned between two electrical contacts located in the floating electrical field area by the dielectrophoretic process.

Figure $4 \mathrm{a}$ shows the transfer characteristics of current $\left(I_{\mathrm{ds}}\right)$ versus applied gate voltage $\left(V_{\mathrm{gs}}\right)$ with different source-drain voltages $\left(V_{\mathrm{ds}}\right)$ for the aligned nanotube shown in the inset, and Figure $4 \mathrm{~b}$ shows its corresponding output characteristics of $I_{\mathrm{ds}}$ versus $V_{\mathrm{ds}}$ for different $V_{\mathrm{gs}}$ from -6 to $0 \mathrm{~V}$. The source-drain current decreases rapidly with increasing gate voltage, which demonstrates the typical p-type transistor characteristics. ${ }^{17}$ This indicates that the nanotube is semiconducting and the transport is dominated by positive carriers (holes). As shown in Figure 


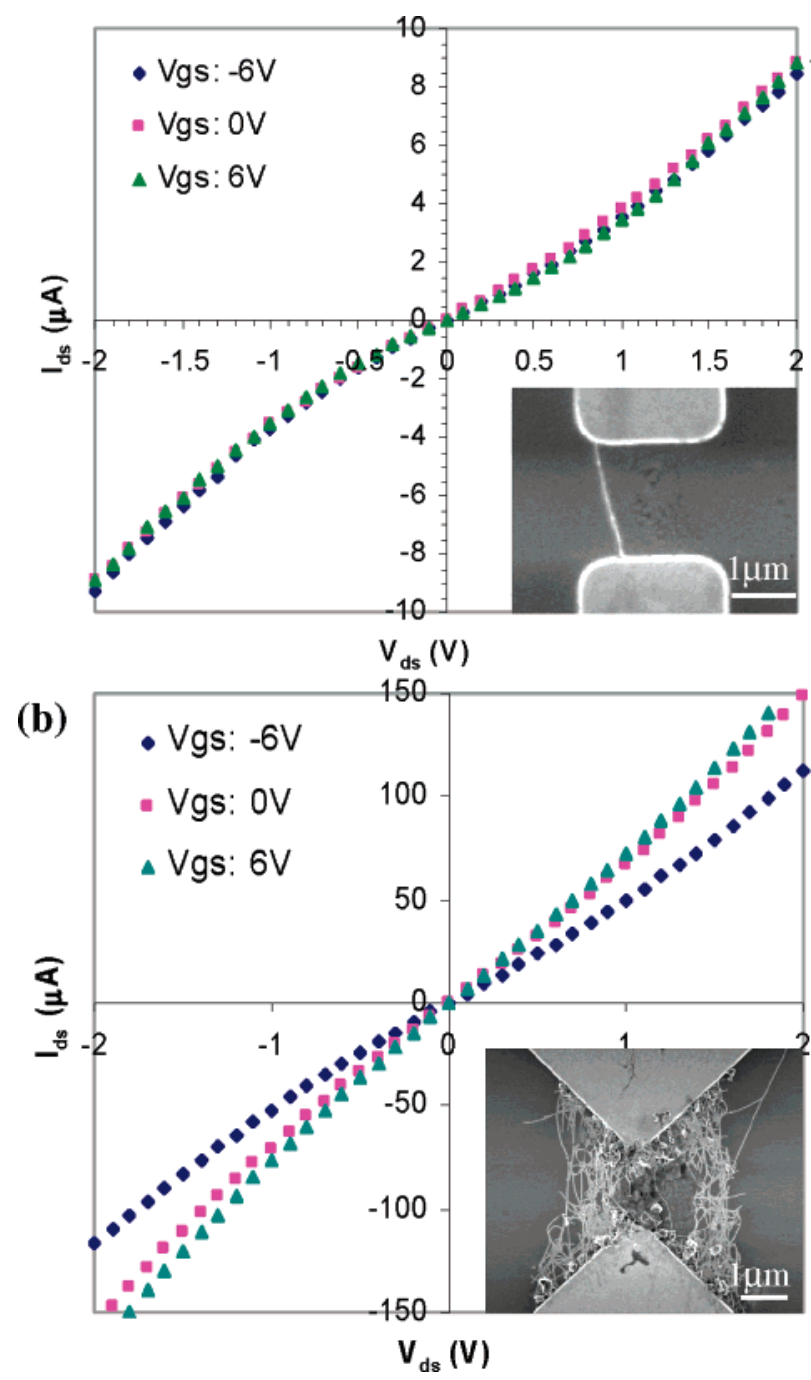

Figure 5. (a) Gate-independent $I-V$ behavior of an individual metallic nanotube. (Inset: SEM image of the nanotube.) (b) Gate-dependent $I-V$ behavior of nanotube bundles consisting of both semiconducting and metallic nanotubes at the gate voltages of $-6,0$, and $6 \mathrm{~V}$. (Inset: SEM image of the nanotube bundles.)

TABLE 1: Comparison of Device Characteristics of Three Kinds of Back-Gated Nanotube Transistors

\begin{tabular}{|c|c|c|c|c|c|}
\hline $\begin{array}{l}\text { contact } \\
\text { materials }\end{array}$ & $\begin{array}{l}\text { channel } \\
\text { length } \\
(\mathrm{nm})\end{array}$ & $\begin{array}{c}\text { gate } \\
\text { oxide } \\
\text { thickness } \\
(\mathrm{nm})\end{array}$ & $\begin{array}{c}\text { inverse } \\
\text { subthreshold } \\
\text { slope } \\
\text { (mV/decade) }\end{array}$ & $\begin{array}{l}\text { transconductance } \\
\qquad(\mu \mathrm{S})\end{array}$ & references \\
\hline & & & 2500 & & \\
\hline $\mathrm{TiC}$ & 800 & 150 & 730 & $0.280\left(V_{\mathrm{ds}}=1.0 \mathrm{~V}\right)$ & 18 and 19 \\
\hline platinum & 2500 & 1000 & 1200 & $0.303\left(V_{\mathrm{ds}}=1.5 \mathrm{~V}\right)$ & this work \\
\hline
\end{tabular}

4a, the device turns on nicely, where the threshold voltage $V_{\text {th }}$ (the gate voltage for which the device starts to turn on) is approximately $1.5 \mathrm{~V}$, and the on-off ratio is about $10^{5}$. The inverse subthreshold slope, $S=\mathrm{d} V_{\mathrm{gs}} / \mathrm{d}\left(\log I_{\mathrm{ds}}\right)$, a measure of the efficiency of the gate field in turning on the device, is 1200 $\mathrm{mV} /$ decade, and the transconductance, $g_{\mathrm{m}}=\mathrm{d} I_{\mathrm{ds}} / \mathrm{d} V_{\mathrm{gs}}$, is about $0.3 \mu \mathrm{S}$. The device characteristics, such as the steepness of the subthreshold slope, are consistent with the behavior of a singlenanotube device reported previously, which further verifies that the nanotube is an individual nanotube instead of a small bundle of nanotubes. ${ }^{18,19}$ As listed in Table 1, the measured data of our individual nanotube transistors are comparable to the performance achieved by other back-gated nanotube transistors prepared using other methods, where nanotubes were first randomly dispersed onto a thermal oxide layer formed on a silicon wafer and then the contact materials were deposited on top of the two ends of selected nanotubes. ${ }^{18,19}$ By comparison, it is predictable that once the thickness of the gate oxide is reduced or a gate with a high dielectric constant is utilized, the performance of dielectrophoretically fabricated nanotube transistors will be improved. This prediction is also supported by the theoretical calculations and experimental results of nanotube transistors fabricated by other methods. ${ }^{20-22}$

Figure 5a shows the curves of drain current versus sourcedrain voltage of the nanotube illustrated in the inset of Figure 5 a for different gate voltages at $-6,0$, and $6 \mathrm{~V}$. The drain currents are independent of the gate voltage, which indicates that the gate voltage does not affect the carrier transport and, therefore, the tube is metallic. As a result, this suggests that well-aligned individual semiconducting nanotubes as well as individual metallic nanotubes can be assembled between two floating electrodes.

To compare the output properties of a single carbon nanotube and a bundle of nanotubes, we conducted the measurement on a nanotube transistor made of bundles of SWCNTs as shown in the insert in Figure 5b. Note in Figure 5b that the drain currents are influenced by applied gate voltages. The current increases as the gate voltage changes from -6 to $6 \mathrm{~V}$, which suggests there are some semiconducting nanotubes. When the gate voltage is turned off $\left(V_{\mathrm{gs}}=0 \mathrm{~V}\right)$, there is still a fairly high drain current linearly proportional to the applied drain voltage, which means there are some metallic nanotubes as well. Note that at $V_{\mathrm{gs}}=0 \mathrm{~V}$ the current magnitude is about 15 times larger than that of the single nanotube in Figure 5a. This suggests that it would be misleading to collect electrical data without revealing the structural configuration of the nanotubes in the devices and assume that the dielectrophoresis process could separate metallic and semiconductor nanotubes from their admixtures. Our study demonstrates that this assumption is true only if individual nanotube alignments (most of them were found in the floating electrode areas) were achieved. Therefore, this study demonstrates a very promising method for fabricating semiconductor nanotube transistors and provides significant evidence for further understanding of the intrinsic mechanism of dielectrophoresis separation and displacement of carbon nanotubes. A process for positioning selected types of nanotubes from an admixture, such as exclusively semiconducting or metallic nanotubes, is developing through parameter optimizations, including electrode configuration, frequency, and dispersion solution medium.

\section{Conclusions}

In summary, our experimental results show that the magnitude of the dielectrophoresis force does not only depend on the electrical field gradient but is also proportional to the volume of solids in the suspension. When a droplet of nanotube suspension was applied onto a substrate with arrays of patterned electrodes, bundles of nanotubes and other impurities were first selectively moved onto the region with the electrical field of the largest gradient, which was located between two control electrodes. Then, individual nanotubes with a high purity were straightened and positioned between floating electrodes. The yield for the alignment of individual SWCNTs is about $26 \%$. Furthermore, the fabricated nanotube transistors demonstrate excellent electrical characteristics including turn on field, onoff ratio, inverse subthreshold slope, and transconductance. Therefore, this study further advances the effectiveness of using the dielectrophoresis method for the separation, purification, and site-selective alignment of individual nanotubes between two electrical contacts. These findings are significant for the fabrication of nanotube transistors and other nanotube devices. 
Acknowledgment. This research was partially supported by the Intel Corporation and the National Science Foundation (DMR-220926 and ECS-0217061). The authors thank Dr. Raj Solanki and Cheng Qi for their gracious help with the use of electrical characterization facilities and James Hedberg, Chiaching Pan, Matthew C. Crews, Michael Coulter, and Logan Love for their discussions and involvement in parts of these experiments.

\section{References and Notes}

(1) Tans, S.; Verschueren, S.; Dekker, C. Nature 1998, 393, 49.

(2) Javey, A.; Guo, J.; Wang, Q.; Lundstrom, M.; Dai, H. J. Nature 2003, 424, 654 .

(3) Sazonova, V.; Yaish, Y.; Üstünel, H.; Roundy, D.; Arias, T. A.; McEuen, P. L. Nature 2004, 431, 284.

(4) Mason, N.; Biercuk, M. J.; Marcus, C. M. Science 2004, 303, 655.

(5) Dong, L. F.; Jiao, J.; Pan, C. C.; Tuggle, D. W. Appl. Phys. A 2004, 78, 9 .

(6) Chen, X. Q.; Saito, T.; Yamada, H.; Matsushige, K. Appl. Phys. Lett. 2001, 78, 3714.

(7) Krupke, R.; Hennrich, F.; Löhneysen, H. v.; Kappes, M. M. Science 2003, 301, 344 .

(8) Krupke, R.; Hennrich, F.; Kappes, M. M.; Löhneysen, H. v. Nano Lett. 2004, 4, 1395.

(9) Chen, Z.; Hu, W. C.; Guo, J.; Saito, K. J. Vac. Sci. Technol., B 2004, 22, 776 . 2263.
(11) Baik, S.; Usrey, M.; Rotkina, L.; Strano, M. S. J. Phys. Chem. B 2004, 108, 15560.

(12) Krupke, R.; Hennrich, F.; Weber, H. B.; Kappes, M. M.; Löhneysen, H. v. Nano Lett. 2003, 3, 1019.

(13) O'Connell, M. J.; Bachilo, S. M.; Huffman, C. B.; Moore, V. C.; Strano, M. S.; Haroz, E. H.; Rialon, K. L.; Boul, P. J.; Noon, W. H.; Kittrell, C.; Ma, J.; Hauge, R. H.; Weisman, R. B.; Smalley, R. E. Science 2002, 297, 593.

(14) Pohl, H. A. Dielectrophoresis; Cambridge University Press: Cambridge, U. K., 1978.

(15) Jones, T. B. Electromechanics of Particles; Cambridge University Press: Cambridge, U. K., 1995.

(16) Brintlinger, T.; Chen, Y. F.; Dürkop, T.; Cobas, E.; Fuhrer, M. S.; Barry, J. D.; Melngailis, J. Appl. Phys. Lett. 2002, 81, 2454.

(17) Homma, Y.; Suzuki, S.; Kobayashi, Y.; Nagase, M.; Takagi, D. Appl. Phys. Lett. 2004, 84, 1750 .

(18) Martel, R.; Derycke, V.; Lavoie, C.; Appenzeller, J.; Chan, K. K.; Tersoff, J.; Avouris, Ph. Phys. Rev. Lett. 2001, 87, 256805.

(19) Martel, R.; Derycke, V.; Appenzeller, J.; Wind, S.; Avouris, Ph. In Design Automation Conference Proceedings, 39th Design Automation Conference; Association for Computing Machinery: New York, 2002; p 94.

(20) Bachtold, A.; Hadley, P.; Nakashini, T.; Dekker, C. Science 2001, 294, 1317.

(21) Heinze, S.; Tersoff, J.; Martel, R.; Derycke, V.; Appenzeller, J.; Avouris, Ph. Phys. Rev. Lett. 2002, 89, 106801.

(22) Javey, A.; Kim, H.; Brink, M.; Wang, Q.; Ural, A.; Guo, J.; McIntyre, P.; McEuen, P.; Lundstrom, M.; Dai, H. J. Nat. Mater. 2002, 1, 241. 